Pacific

Journal of

Mathematics

\title{
ON INVARIANTS FOR LEGENDRIAN KNOTS
}

ANDRÁS I. STIPSICZ AND VERA VÉRTESI 


\title{
ON INVARIANTS FOR LEGENDRIAN KNOTS
}

\author{
ANDRÁS I. STIPSICZ AND VERA VÉRTESI
}

\begin{abstract}
Let $(Y, \xi)$ be a contact 3-manifold and $L$ a null-homologous Legendrian knot in it. We determine the connection between the sutured invariant $\mathbf{E H}(L)=\mathbf{E H}\left(Y-v(L),\left.\xi\right|_{Y-v(L)}\right)$ of $L$ and the Legendrian invariant $\widehat{\mathscr{L}}(L)$ defined in a paper by Lisca, Ozsváth, Stipsicz and Szabó. We derive a vanishing theorem for $\widehat{\mathscr{L}}(L)$ in the presence of Giroux torsion in the complement of the knot, and reprove several known properties of the Legendrian invariant from this perspective.
\end{abstract}

\section{Introduction}

A knot $L$ in a closed, contact 3-manifold $(Y, \xi)$ is Legendrian if the tangent vectors of the knot are contained by the contact 2-plane field $\xi$. The knot $T$ is transverse, if the (nonzero) tangent vectors are not contained by $\xi$. Legendrian and transverse knot theory has been shaped by advances in convex surface theory [Etnyre and Honda 2001] (showing that different looking objects are actually equivalent) and by the introduction of various invariants of these knots - proving that different looking objects are, in fact, different. Examples of such invariants are provided by Chekanov's differential graded algebras [Chekanov 2002] and contact homology [Eliashberg 1998]. More recently, Heegaard Floer homology provided various sets of invariants: for knots in the standard contact 3-sphere the combinatorial construction of knot Floer homology through grid diagrams [Ng et al. 2007; Ozsváth et al. 2008], for null-homologous knots in general contact 3-manifolds the Legendrian invariant of [Lisca et al. 2008] and for general Legendrian knots the sutured invariant of the knot complement [Honda et al. 2007].

The aim of this paper is to set up a relation between these last two invariants. To set the stage, recall that the Legendrian invariant $\widehat{\mathscr{L}}(L)$ of the null-homologous Legendrian knot $L \subset(Y, \xi)$ defined in [Lisca et al. 2008] takes its value in the knot Floer homology group $\widehat{\mathrm{HFK}}(-Y, L)$. (The theory admits a version where the

MSC2000: primary 57M50; secondary 53C15.

Keywords: Legendrian and transverse knot, Heegaard Floer homology, sutured Floer homology. AS acknowledges support from the Clay Mathematics Institute. AS was also partially supported by OTKA 49449 and by Marie Curie TOK project BudAlgGeo. VV was supported by NSF grant number FRG-0244663 and OTKA 49449 and 67867. VV was also supported by "Magyar Állami Eötvös Ösztöndíj”. 
invariants are in the more refined group $\mathrm{HFK}^{-}(-Y, L)$, but since the corresponding sutured theory is not developed yet, we will deal only with the $\widehat{\mathrm{HFK}}$-version in this paper.) In turn, the sutured invariant $\operatorname{EH}(L)$ is defined as follows: consider the Legendrian knot $L \subset(Y, \xi)$, and delete a standard neighbourhood $v(L)$ of $L$ with convex boundary. The resulting contact 3-manifold $Y-v(L)$ with convex boundary naturally admits a balanced sutured 3-manifold structure $(Y-v(L), \Gamma)$, and hence by [Juhász 2006] it admits a sutured Floer homology $\operatorname{SFH}(Y-v(L), \Gamma)$. According to [Honda et al. 2007] the contact structure on $Y-v(L)$ specifies an element

$$
\operatorname{EH}(L) \in \operatorname{SFH}(-(Y-v(L)),-\Gamma),
$$

which we will call the sutured invariant of $L$. A relation between sutured Floer homology and knot Floer homology obviously follows from their definitions: suppose that $(Y-v(L), \Gamma)$ is the sutured 3-manifold with toric boundary we get by deleting a neighbourhood of the (not necessarily Legendrian) knot $L$, and $\Gamma$ has two (parallel) components. Then there is an obvious isomorphism

$$
\Psi: \operatorname{SFH}(Y-v(L), \Gamma) \rightarrow \widehat{\operatorname{HFK}}\left(Y_{\Gamma}, L^{\prime}\right)
$$

where $Y_{\Gamma}$ is the Dehn filling of $Y-v(L)$ (and $L^{\prime}$ is the core of the Dehn filling) with slope given by the sutures $\Gamma$. In general, $Y_{\Gamma}$ differs from $Y$ (and therefore $L^{\prime}$ differs from $L$ ). By attaching a specific contact $T^{2} \times[0,1]$ (a basic slice) to $Y-v(L)$, the composition of the map

$$
\Phi: \operatorname{SFH}(-(Y-v(L)),-\Gamma) \rightarrow \operatorname{SFH}\left(-(Y-v(L)), \Gamma^{\prime}\right)
$$

of [Honda et al. 2008] induced by this attachment and the above map $\Psi$ (applied to the suture $\Gamma^{\prime}$ with components isotopic to the meridian of the knot) gives a map

$$
F: \operatorname{SFH}(-(Y-v(L)),-\Gamma) \rightarrow \widehat{\operatorname{HFK}}(-Y, L)
$$

for which we show:

Theorem 1.1. Fix an orientation on the Legendrian knot $L$ and consider one of the basic slices with boundary slopes given by the dividing set of $\partial(Y-v(L))$ on $T^{2} \times\{0\}$ and by the meridian of $L$ on $T^{2} \times\{1\}$. Then the map $F$ defined above maps $\mathrm{EH}(L)$ to $\widehat{\mathscr{L}}(L)$.

A more precise formulation of the theorem will be given in Section 4 after basic slices and orientations have been discussed. A straightforward consequence of the above relation is:

Corollary 1.2. If the complement of a null-homologous Legendrian knot has positive Giroux torsion then $\widehat{\mathscr{L}}(L)$ vanishes. 
Remark 1.3. The same corollary has been found recently by D. S. Vela-Vick [2008] using slightly different arguments.

To put this result in perspective, we recall that a knot type in the standard contact 3 -sphere is called Legendrian simple if two Legendrian knots of the given knot type with identical Thurston-Bennequin and rotation numbers (for definitions of these invariants see [Etnyre 2005]) are Legendrian isotopic. The same notion generalizes to an arbitrary ambient contact 3-manifold $(Y, \xi)$, with a caveat in the case when $\xi$ is overtwisted: in that case Legendrian knots fall into two categories, depending on whether the knot complement is overtwisted (in which case the knot is called loose) or - although $\xi$ is overtwisted - the knot complement is tight (in which case the knot is nonloose or exceptional; see [Eliashberg and Fraser 2008; 1998]). Obviously a loose and a nonloose knot cannot be isotopic. Hence in overtwisted contact 3-manifolds besides the equality of the Thurston-Bennequin and rotation numbers we also require the equality of the looseness of the two knots in defining simplicity. Nonsimple nonloose knots in a variety of overtwisted contact structures have been found in [Lisca et al. 2008]. There is, however, a simple way of constructing nonsimple nonloose knots [Etnyre 2008]: suppose that the knot complement contains an incompressible torus (for example, the knot type is a satellite in $S^{3}$ ) and introduce Giroux torsion along the torus. Since this procedure does not change the homotopy type of the 2-plane field, and $\xi$ is overtwisted by assumption (and overtwisted structures are classified by their homotopy type), after a suitable choice of the knot and the torus we get a Legendrian knot in the same contact 3-manifold with different tight complement. (The verification that the complement remains tight, and that the implementations of different Giroux torsions result in different structures requires delicate arguments [Etnyre 2008].) This method, in fact, can produce infinitely many different Legendrian nonloose knots with the same numerical invariants in these knot types [Etnyre 2008]. We say that $L \subset(Y, \xi)$ is strongly nonloose if $\xi$ is overtwisted and the knot complement is tight with vanishing Giroux torsion. The knot type is strongly nonsimple if there are two strongly nonloose, smoothly isotopic knots with equal numerical invariants which are not Legendrian isotopic. The same simplicity/nonsimplicity definition (with the strong adjective) carries through verbatim for transverse knots (where the role of the numerical invariants is played by the self-linking number of the transverse knot). In this sense, the result of [Lisca et al. 2008] translates to

Corollary 1.4. The knot types of [Lisca et al. 2008, Theorem 1.7 and Corollary 1.8] are strongly nonsimple.

Proof. The distinction of the Legendrian knots $L_{i}$ in [Lisca et al. 2008] went by determining the Legendrian invariants $\widehat{\mathscr{L}}\left(L_{i}\right)$, and since both were nonzero, 
Corollary 1.2 implies that the knots $L_{i}$ are strongly nonloose, concluding the proof.

Notice that in [Ozsváth et al. 2008] the combinatorial theory provided two invariants of $L$ (denoted by $\widehat{\lambda}^{ \pm}(L)$ ), while in [Lisca et al. 2008] the invariant $\widehat{\mathscr{L}}(L)$ depended on an orientation of $L$ - therefore an unoriented Legendrian knot admitted two invariants $\widehat{\mathscr{L}}(L)$ and $\widehat{\mathscr{L}}(-L)$ after an arbitrary orientation of $L$ was fixed. On the other hand, the sutured theory provides a unique element for $L$. The discrepancy is resolved by the observation that the map on sutured Floer homology induced by the basic slice attachment is well-defined only up to a choice: with the given boundary slopes there are two basic slices, and using one the class $\operatorname{EH}(L)$ is transformed into $\widehat{\mathscr{L}}(L)$ while with the other choice the result will be $\widehat{\mathscr{L}}(-L)$ (after an orientation on $L$ is fixed). In order to clarify signs, we reprove a special case of [Lisca et al. 2008, Theorem 7.2] (only in the $\widehat{\mathrm{HFK}}$-theory) regarding the effect of stabilization of $L$ on $\widehat{\mathscr{L}}$ and show:

Theorem 1.5. Let $L$ be an oriented null-homologous Legendrian knot. If $L^{-}$(and $L^{+}$) denotes its negative (respectively positive) stabilization, then

$$
\widehat{\mathscr{L}}\left(L^{-}\right)=\widehat{\mathscr{L}}(L), \quad \text { and } \widehat{\mathscr{L}}\left(L^{+}\right)=0 .
$$

Notice that the invariance of $\widehat{\mathscr{L}}$ under negative stabilization means that, in fact, it is an invariant of the transverse isotopy class of the positive transverse push-off of the Legendrian knot $L$. By this definition the extensions of Corollaries 1.2 and 1.4 to the transverse case are easy exercises. For further results regarding transverse knots using these invariants see [Ng et al. 2007; Ozsváth and Stipsicz 2008]. In fact, in [Ozsváth and Stipsicz 2008] the distinction of various Legendrian and transverse Eliashberg-Chekanov (also know as twist) knots and 2-bridge knots was carried out by computing their $\widehat{\mathscr{L}}$-invariants. As a corollary, Theorem 1.1 readily implies:

Corollary 1.6. The complement of the Eliashberg-Chekanov knot $E_{n}$ (which is the 2-bridge knot of type $\frac{2 n+1}{2}$ ) for odd $n$ admits at least $\left\lceil\frac{n}{4}\right\rceil$ different tight contact structures (distinguished by the sutured invariant) with convex boundary and dividing set $\Gamma$ of two components with slope 1.

Performing contact (-1)-surgery along a Legendrian knot $L$ gives a well-defined contact structure $\xi_{-1}$ on the surgered 3-manifold $Y_{-1}$. The core $L^{\prime}$ of the glued-back solid torus is a Legendrian knot in $\left(Y_{-1}, \xi_{-1}\right)$. Suppose that $L^{\prime}$ is null-homologous in $Y_{-1}$. Using the sutured invariant we deduce:

Theorem 1.7. Under the circumstance described above,

$$
\widehat{\mathscr{L}}(L) \neq 0 \Longrightarrow \widehat{\mathscr{L}}\left(L^{\prime}\right) \neq 0 .
$$


The paper is organized as follows. In Section 2 we review the basic definitions we need about contact structures. Section 3 gives a short description of sutured Heegaard Floer homology and the definition of the Legendrian invariants. In Section 4 we state a precise version of Theorem 1.1 and prove it together with the consequences given in Section 1.

\section{Contact preliminaries}

2A. Contact 3-manifolds. A surface $\Sigma$ in the contact 3-manifold $(Y, \xi)$ is convex if there is a contact vector field $X$ defined near $\Sigma$ which is transverse to $\Sigma$. The set of points $p \in \Sigma$ where $X_{p} \in \xi_{p}$ is usually denoted by $\Gamma$ and called the dividing set of the convex surface $\Sigma$. It turns out that $\Gamma$ is an embedded 1-manifold, partitioning $\Sigma$ into $\Sigma_{+}$and $\Sigma_{-}$, and the contact structure $\xi$ is determined by $\Gamma$ near $\Sigma$. For a more complete treatment of the subject, see [Etnyre 2005; Etnyre and Honda 2001; Ozbagci and Stipsicz 2004].

Suppose that $L$ is an oriented null-homologous Legendrian knot in the contact 3-manifold $(Y, \xi)$. Let $S$ be a Seifert surface of $L$ in convex position. Orient $S$ such that its boundary orientation gives the orientation for $L$. The rotation number then can be computed as

$$
\operatorname{rot}(L)=\chi\left(S_{+}\right)-\chi\left(S_{-}\right) .
$$

Define the negative and positive stabilizations $L^{-}$and $L^{+}$by modifying $L$ near a point as it is depicted by Figure 1 . The effect of a positive (respectively negative) stabilization on the numerical invariants of $L$ can be easily computed as

$$
\operatorname{tb}\left(L^{ \pm}\right)=\operatorname{tb}(L)-1 \quad \text { and } \quad \operatorname{rot}\left(L^{ \pm}\right)=\operatorname{rot}(L) \pm 1 .
$$

Notice that the sign of the stabilization makes sense only after fixing an orientation for the Legendrian knot.

2B. Sutured 3-manifolds. A sutured 3-manifold is a pair $(Y, \gamma)$ where $Y$ is a compact, oriented 3-manifold with boundary and $\gamma \subset \partial Y$ is a disjoint set of embedded tori and annuli. Every component of $R(\gamma)=\partial Y-\gamma$ is oriented, and $R_{+}$(respectively $R_{-}$) is the union of those components where the normal vector points out (respectively in) $Y$. The sutured manifold is called balanced if all sutures are

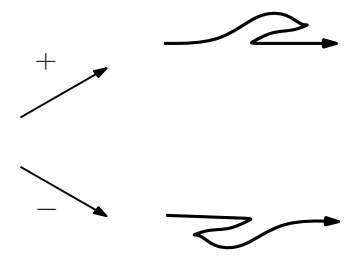

Figure 1. Positive and negative stabilization. 
annular, $Y$ has no closed components, every boundary component admits a suture and $\chi\left(R_{+}\right)=\chi\left(R_{-}\right)$on every component of $Y$. As is customary, annular sutures are symbolized by the homologically nontrivial simple closed curves they contain, the collection of which is denoted by $\Gamma$. Without confusion, the term "suture" will also refer to these curves, and sometimes to their union $\Gamma$. The suture $\Gamma$ is oriented as the boundary of $R_{+} \subset \partial Y$. We will consider only balanced sutured manifolds in this paper.

2C. Partial open books. Partial open books are generalizations of open books for 3-manifolds with boundary. This notion was introduced by Honda, Kazez and Matić [2007]; see also [Etgü and Ozbagci 2007; 2008].

Definition 2.1. An abstract partial open book is a triple $(S, P, h)$ where $S$ is a connected surface with boundary, $P$ is a proper subsurface of $S$ which is a union of 1-handles attached to $S-P$, and $h: P \rightarrow S$ is an embedding that restricts to the identity near the boundary $\partial P \cap \partial S$.

A partial open book defines a 3-manifold $Y$ with boundary as follows. First construct the handle-body $S \times[-1,0] / \sim$ and the compression-body $P \times[0,1] / \sim$, where $(x, t) \sim\left(x, t^{\prime}\right)$ for $x \in \partial S$ and $t, t^{\prime} \in[-1,1]$. (Note that on $P \times[0,1]$ we just contract the points with first coordinate in $\partial P \cap \partial S$.) Then glue them together with the maps $P \times\{0\} \hookrightarrow S \times\{0\}$ and $h: P \times\{1\} \rightarrow S \times\{-1\}$. A schematic picture of $Y$ is given by Figure 2. The resulting 3-manifold naturally carries the structure of a balanced sutured manifold: take

$$
\Gamma=\overline{\partial S-\partial P} \times\left\{-\frac{1}{2}\right\} \cup-\overline{(\partial P-\partial S)} \times\left\{\frac{1}{2}\right\} \subset \partial Y .
$$

Now $R_{+}=\overline{S-P} \times\{0\}, R_{-}=\overline{S-h(P)} \times\{-1\}$; consequently $\chi\left(R_{+}\right)=\chi\left(R_{-}\right)$ follows at once.

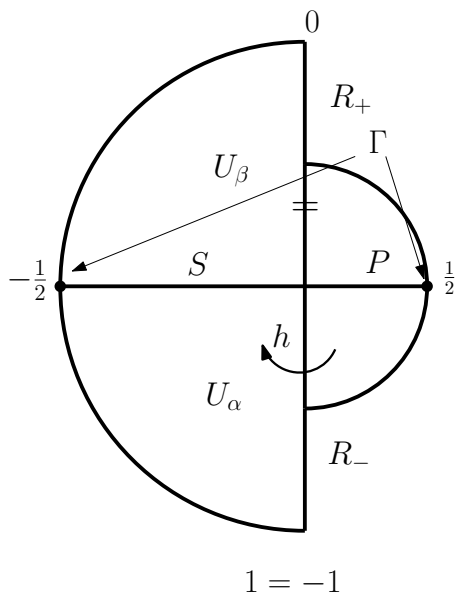

Figure 2. Schematic picture of a partial open book decomposition. 
Both the handle-body $S \times[-1,0] / \sim$ and the compression-body $P \times[0,1] / \sim$ admit unique tight contact structures with convex boundary and dividing set $\partial S$ (and $\partial P$, respectively); see [Etgü and Ozbagci 2008; Torisu 2000]. As the dividing sets match up, we can glue these contact structures to obtain a contact structure $\xi$ on $Y$ with dividing set $\Gamma$ on the convex boundary $\partial Y$. In this sense a partial open book decomposition determines a contact structure with convex boundary (inducing the dividing set given by the sutures associated to the partial open book).

The partial open book decomposition naturally induces a Heegaard decomposition of $Y$ with the compression-bodies

$$
U_{\alpha}=P \times\left[\frac{1}{2}, 1\right] \cup S \times\left[-1,-\frac{1}{2}\right] \quad \text { and } \quad U_{\beta}=S \times\left[-\frac{1}{2}, 0\right] \cup P \times\left[0, \frac{1}{2}\right],
$$

divided by the Heegaard surface

$$
\Sigma=\partial U_{\alpha}=S \times\left\{-\frac{1}{2}\right\} \cup-P \times\left\{\frac{1}{2}\right\} .
$$

Consistently with the sutured 3-manifold structure, the boundary of $U_{\alpha}$ (and $U_{\beta}$, respectively) consists of the union of $\Sigma$ (respectively $-\Sigma$ ), $R_{-}$(respectively $R_{+}$) and a collar neighbourhood for $\Gamma$; furthermore

$$
\Gamma=\partial \Sigma\left(=\partial R_{+}=-\partial R_{-}\right) .
$$

Every contact 3-manifold with convex boundary $(Y, \xi)$ admits a partial open book decomposition that is compatible with $\xi$ in the above sense [Honda et al. 2007]. To see this, consider a contact cell-decomposition for $Y$ whose 1-skeleton $C$ is a direct product near the boundary $\partial Y$ and intersects the boundary on the dividing set. As Legendrian arcs have standard neighbourhood, there is a neighbourhood $v(C)$ of $C$ with convex boundary and with dividing curves of two components. The dividing curve separates $-\partial v(C)$ into a positive and a negative part $(-\partial v(C))_{+}$ and $(-\partial v(C))_{-}$. Setting $P=(-\partial v(C))_{+}$the neighbourhood $v(C)$ can be written as $P \times[0,1] / \sim$. As $C$ was the 1 -skeleton of a contact cell-decomposition, $Y-$ $v(C)$ is product disk-decomposable: it is divided by the 2-cells of the contact cell-decomposition (that are disks with $\mathrm{tb}=-1$ ) to a union of tight contact 3balls. Thus for $S=\partial(Y-v(C))_{+}$the handle-body $Y-v(C)$ can be written as $Y-v(C)=S \times[-1,0] / \sim$, and

$$
P=(-\partial v(C))_{+} \subseteq(\partial(Y-v(C)))_{+}=S .
$$

Note that by construction $\left.\xi\right|_{Y-v(C)}$ is tight, its boundary $\partial(Y-v(C))$ is convex, and the dividing set $\Gamma_{\partial(Y-v(C))}$ is isotopic to $\partial S \times\{0\}$.

2D. Bypass attachment. Next we review the change of the partial open book decomposition after a bypass is attached along a Legendrian curve $c$ on the boundary. 
For a complete discussion of bypass attachments see [Honda 2000]. The considerations below already appeared in [Honda et al. 2007, Example 5].

Let $(Y, \partial Y, \xi)$ be a contact 3-manifold with convex boundary. Suppose that we are given a Legendrian arc $c \subset \partial Y$ that starts and ends on the dividing set $\Gamma_{\partial Y}$ and intersects $\Gamma_{\partial Y}$ in one additional point. Attaching a bypass along $c$ is roughly speaking - the attachment of the neighbourhood of a "half overtwisted disk". This is a disk $D$ with boundary $\partial D=c \cup d$, where $\partial D \cap \partial Y=c$, and the dividing curve on $D$ consists of a single arc with both of its endpoints on $c$. The resulting manifold is diffeomorphic to $Y$ with contact structure $\xi^{c}$, and the dividing curve $\Gamma$ is changed in the neighbourhood of $c$ to $\Gamma^{c}$ as it is shown on Figure 3.

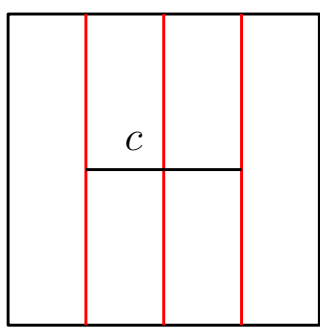

$\Gamma$
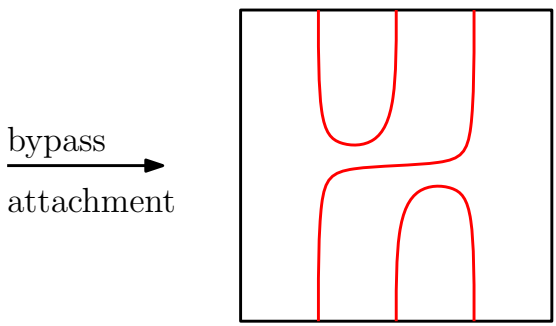

$\Gamma^{c}$

Figure 3. Bypass attachment.

Take a partial open book decomposition for $(Y, \partial Y, \xi)$ coming from a contact cell-decomposition whose 1-skeleton $C$ misses the attaching $\operatorname{arc} c$. Let $c_{ \pm}=c \cap R_{ \pm}$. Under the identification of $Y-v(C)$ with $S \times[-1,0] / \sim$, both $c_{+}$and $c_{-}$are arcs on $S$. The bypass attachment can be thought of consisting of a 1-handle attachment with core $d$ followed by a canceling 2-handle attachment along the curve $a=$ $a_{+} \cup a_{-}$of Figure 4. The contact cell-decomposition can be extended to the new manifold $\left(Y^{\prime}, \xi^{\prime}\right)$ (where $Y^{\prime}$ is, in fact, diffeomorphic to $Y$ ) by including the cocore

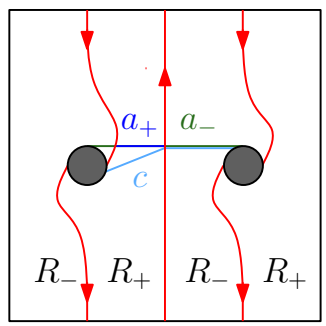

Figure 4. The grey areas indicate the attaching regions of the 1handle. The attaching curve for the 2-handle is $a=a_{+} \cup a_{-}$and $a_{-}$ is assumed to go parallel to the core of the 1-handle in the negative region. 
of the 2-handle in the 1-skeleton. Thus

$$
C^{\prime}=C \cup(\text { cocore of the 2-handle) }
$$

and the page $S^{\prime}$ of the partial open book decomposition resulting from this contact cell-decomposition is $S \cup(-\partial v(d))_{+}=S \cup$ (1-handle). Denote the intersection of the attaching circle of the canceling 2-handle with the positive and negative parts of $\partial(Y \cup\{1$-handle $\})$ by $a_{ \pm}=a \cap R_{ \pm}^{\prime}$. As depicted in Figure 4, the arc $a_{+}$can be pushed off to lie entirely on the boundary of the old manifold $Y$, thus $a_{+} \subset R_{+}$. Note that $c_{+}$and $a_{+}$are isotopic. They have one endpoint that agrees with the endpoint of both $c_{-}$and $a_{-}$, and the other one is moved in the direction given on $\Gamma_{\partial Y}$ as the boundary of $R_{+}$. These curves can again be thought of as being on $S$. Now

$$
R_{+}^{\prime}=\left(R_{+}-v\left(a_{+}\right)\right) \cup(\partial(-v(d)))_{+},
$$

thus $P^{\prime}=P \cup v\left(a_{+}\right)$. The monodromy $h^{\prime}$ remains the same on $P$, so we only need to understand it on $a_{+}$. To push $a_{+}$through $v(C)$ we just have to push it through the newly attached 1-handle, so $h^{\prime}\left(a_{+}\right)=a_{-}$. The arc $a_{-}$can be split to two subarcs $a_{-} \cap S$ and the core of the 1-handle in $S^{\prime}$.

2E. Basic slices. We give a short description of basic slices defined by Honda [2000]. Suppose that $\xi$ is a contact structure on $T^{2} \times[0,1]$ with convex boundary and with two-component dividing curves on each of its boundary components. The dividing curves are homotopically nontrivial and parallel. Fix a trivialization for $T^{2}$ as $\mathbb{R}^{2} / \mathbb{Z}^{2}$ and let $s_{i}$ denote the slope of the dividing curves on $T^{2} \times\{i\}$ $(i \in\{0,1\})$. The contact 3 -manifold $\left(T^{2} \times[0,1], \xi\right)$ is called minimally twisting if every convex torus parallel to the boundary has slope $s$ in $\left[s_{1}, s_{0}\right]$. (By $\left[s_{1}, s_{0}\right]$ we mean $\left[s_{1}, \infty\right] \cup\left[-\infty, s_{0}\right]$ if $s_{1} \geq s_{0}$.) A basic slice is a minimally twisting tight contact structure $\left(T^{2} \times[0,1], \xi\right)$, with convex boundary and with two dividing curves on each $T^{2} \times\{i\}$ and boundary slopes $s_{0}$ and $s_{1}$ forming an integral basis for $\mathbb{Z}^{2}$. For fixed boundary conditions (up to isotopy) there are two basic slices distinguished by their relative Euler class, which differ by their sign; there is no canonical positive or negative choice.

One way to obtain a basic slice is by gluing a bypass to an $I$-invariant neighbourhood of a convex $T^{2}$ with two dividing curves. For a given slope of the attaching curve there are two ways of attaching a bypass corresponding to the two different basic slices; see Figure 6 . Any basic slice can be obtained by this construction.

Suppose that $\left(T^{2} \times[0,1], \xi_{0}\right)$ and $\left(T^{2} \times[1,2], \xi_{1}\right)$ are basic slices with boundary slopes $s_{i}$ on $T^{2} \times\{i\} \quad(i \in\{0,1,2\})$. As the dividing curves match up on $T^{2} \times\{1\}$, we can glue them together to obtain $\left(T^{2} \times[0,2], \xi=\xi_{0} \cup \xi_{1}\right)$. If in addition we require that the shortest representatives of $s_{0}$ and $s_{2}$ give an integral basis for $\mathbb{Z}^{2}$ and $\left[s_{0}, s_{1}\right] \cup\left[s_{1}, s_{2}\right] \neq[-\infty, \infty]$, then $\left(T^{2} \times[0,2], \xi\right)$ is minimally twisting. It is either 
overtwisted or a single basic slice depending on whether the basic slices $\left(T^{2} \times\right.$ $\left.[0,1], \xi_{0}\right)$ and $\left(T^{2} \times[1,2], \xi_{1}\right)$ have the same or opposite signs. Note that "having the same sign" makes sense in this setting, once we require the trivialization of $\xi_{0}$ and $\xi_{1}$ to agree over $T^{2} \times\{1\}$.

\section{Heegaard Floer invariants}

In [Ozsváth and Szabó 2004c; 2004b] invariants of closed, oriented 3-manifolds have been introduced. In the simplest version, these invariants are given as follows. Suppose that the 3 -manifold $Y$ is given by a Heegaard diagram $\left(\Sigma_{g}, \boldsymbol{\alpha}, \boldsymbol{\beta}\right)$, where $\Sigma_{g}$ is a genus- $g$ surface, the $g \alpha$-curves $\alpha=\left\{\alpha_{1}, \ldots, \alpha_{g}\right\}$ correspond to belt circles of 1-handles, while the $g \beta$-curves $\beta=\left\{\beta_{1}, \ldots, \beta_{g}\right\}$ to attaching circles of 2-handles in a handle decomposition of $Y$ with a unique 0 - and 3-handle. In particular, the $\alpha$ - (and similarly the $\beta$-) circles are disjoint, and linearly independent in homology. By fixing a base point $w \in \Sigma_{g}$ in the complement of all the $\alpha$ - and $\beta$ curves, the chain complex $(\widehat{\mathrm{CF}}(Y), \partial)$ is defined as follows: consider the $\mathbb{Z}_{2}$-vector space $\widehat{\mathrm{CF}}(Y)$ freely generated by the intersections

$$
\mathbb{T}_{\alpha} \cap \mathbb{T}_{\beta} \subset \operatorname{Sym}^{g}\left(\Sigma_{g}\right),
$$

where the tori $\mathbb{T}_{\alpha}$ and $\mathbb{T}_{\beta}$ are the products of the $\alpha$ - and $\beta$-curves, respectively. The boundary operator $\partial$ is defined by counting holomorphic disks in $\operatorname{Sym}^{g}\left(\Sigma_{g}\right)$ (for an appropriate choice of almost complex structure) connecting intersection points of $\mathbb{T}_{\alpha}$ and $\mathbb{T}_{\beta}$, which avoid the divisor $V_{w}=\{w\} \times \operatorname{Sym}^{g-1}\left(\Sigma_{g}\right)$. If $\left(\Sigma_{g}, \boldsymbol{\alpha}, \boldsymbol{\beta}\right)$ satisfy the technical condition of admissibility (which can always be arranged by suitable isotopies; see [Ozsváth and Szabó 2004b]) then the homology $\widehat{\mathrm{HF}}(Y)$ of the resulting chain complex is a diffeomorphism invariant of $Y$.

Variants of this construction provide invariants for knots and for sutured 3manifolds, as will be outlined below. First, the choice of another point $z \in \Sigma_{g}$ in the complement of the $\alpha$ - and the $\beta$-curves determines a knot $K \subset Y$, and by taking $\widehat{\mathrm{CFK}}(Y, K)=\widehat{\mathrm{CF}}(Y)$ and modifying $\partial$ to $\partial_{K}$ by only allowing holomorphic disks avoiding both $V_{w}$ and $V_{z}$ we get a chain complex $\left(\widehat{\mathrm{CFK}}(Y, K), \partial_{K}\right)$, with homology the knot Floer homology group $\widehat{\operatorname{HFK}}(Y, K)$. As it is shown in [Ozsváth and Szabó 2004a; Rasmussen 2003], for $K$ null-homologous in $Y$ this homology group will be an invariant of the pair $(Y, K)$.

Suppose now that $\Sigma$ is a compact surface with nonempty boundary. Then by fixing $k$ linearly independent (in homology) and disjoint $\alpha$ - (and similar $\beta$-) circles, the attachment of the appropriate handles gives a balanced sutured 3-manifold with sutures being equal to $\partial \Sigma$. In fact, every balanced sutured 3-manifold arises in this way. The previous scheme applies verbatim (without even the choice of base points) and provides a chain complex $\left(\operatorname{SFC}(Y, \Gamma), \partial_{\Gamma}\right)$, ultimately defining 
the sutured Floer homology group $\operatorname{SFH}(Y, \Gamma)$, which has been shown to be an invariant of the sutured 3-manifold [Juhász 2006].

If $\Sigma$ has exactly two boundary components and $\bar{\Sigma}$ denotes the capped-off closed surface, and if the number of attaching curves $k$ equals to the genus of $\Sigma$ and the curves are homologically independent in $\bar{\Sigma}$, then the corresponding sutured 3-manifold has toric boundary with a 2-component suture, and by placing two marked points on the caps we get an identification

$$
\Psi: \operatorname{SFH}(Y, \Gamma) \rightarrow \widehat{\operatorname{HFK}}\left(Y_{\Gamma}, L^{\prime}\right),
$$

where $Y_{\Gamma}$ is the result of Dehn filling of $Y$ with slope given by a component of $\Gamma$ and $L^{\prime}$ is the core of the glued-up solid torus.

The contact invariant. Suppose that $(Y, \xi)$ is a contact 3-manifold with convex boundary, and consider a partial open book compatible with $\xi$. Let $\left\{b_{1}, \ldots, b_{k}\right\}$ be a basis for $H_{1}(P, \partial S \cap \partial P)$. The disks swept out by the $b_{i}$ 's in the $U_{\beta}$ handle-body have boundaries $\beta_{i}=b_{i} \times\left\{\frac{1}{2}\right\} \cup b_{i} \times\left\{-\frac{1}{2}\right\}$. Isotope each $b_{i}$ to an arc $a_{i}$ that intersects it transversely in a single point, and whose endpoints are moved in the direction given by the boundary orientation of $-P$. In the $U_{\alpha}$ handle-body $a_{i}$ sweeps out a disk with boundary $\alpha_{i}=a_{i} \times\left\{\frac{1}{2}\right\} \cup h\left(a_{i}\right) \times\left\{-\frac{1}{2}\right\}$, providing a Heegaard diagram $(\Sigma, \boldsymbol{\alpha}, \boldsymbol{\beta})$ for $(Y, \Gamma)$. The single intersection point $\mathbf{y}=\left(a_{i} \cap b_{i}\right)$ on $P \times\left\{\frac{1}{2}\right\}$ can be shown to represent a cycle in $\operatorname{SFC}(-\Sigma, \boldsymbol{\alpha}, \boldsymbol{\beta})$, thus it defines an element $\operatorname{EH}(Y, \xi)$ in $\operatorname{SFH}(-Y,-\Gamma)$. (Notice the orientation reversal of the Heegaard surface $\Sigma$.) As has been proven by Honda, Kazez and Matić [2007], this element is independent of the choices made throughout its definition and gives the invariant $\operatorname{EH}(Y, \xi)$ of the contact structure $(Y, \xi)$. In the special case when the contact 3-manifold with convex boundary is given as the complement of a standard neighbourhood of a Legendrian knot in a closed contact 3-manifold $(Y, \xi)$, the resulting element will be denoted by $\operatorname{EH}(L)$. Note that by the Legendrian Neighbourhood Theorem, in this case $\Gamma$ consists of two parallel simple closed curves in $\partial(Y-v(L))$.

The Legendrian invariant. Now consider an oriented, null-homologous Legendrian knot in the closed contact 3-manifold $(Y, \xi)$. There is an open book decomposition of $Y$ compatible with $\xi$ containing $L$ on one of its pages $S=S \times\left\{\frac{1}{2}\right\}$. Consider a properly embedded $\operatorname{arc} b_{1}$ in $S$ intersecting $L$ exactly once. The disk $b_{1} \times[0,1]$ is a meridional disk for $L$. Orient $b_{1}$ so that the boundary orientation of $\partial\left(b_{1} \times[0,1]\right)=-b_{1} \times\{0\} \cup b_{1} \times\{1\}$ agrees with the natural orientation of the meridian for $L$. (Such an oriented arc $b_{1}$ will be called a half-meridian of L.) With these conventions the orientation of $S$ coincides with the orientation induced by $\left(b_{1}, L\right)$. Our setup here will be slightly different from the one used in [Lisca et al. 2008], but the resulting Heegaard diagram and the element specified in it will be actually the same already on the chain-level. 


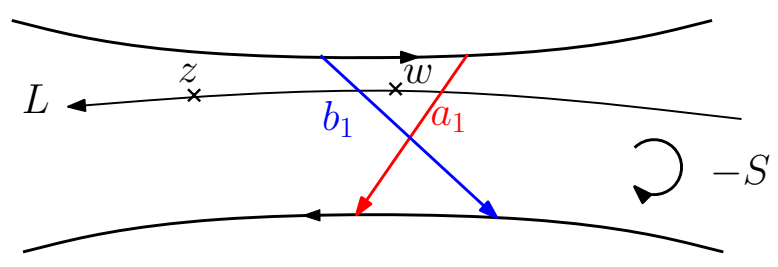

Figure 5. The placement of the basepoints.

Pick a basis $\left\{b_{1}, \ldots, b_{g}\right\}$ of $H_{1}(S, \partial S)$ such that $b_{1}$ is a half-meridian of $L$. Isotope all the $b_{i}$ 's to $a_{i}$ 's as before and place the basepoint $z$ in the "big" region that is not swept out by the isotopies of the $b_{i}$, and put $w$ between $b_{1}$ and $a_{1}$. This can be done in two essentially different ways, and exactly one of them corresponds to the chosen orientation of $L$. If $b_{1}$ is oriented as described above, $w$ should be placed close to the tail of $b_{1}$; see Figure 5 . The single intersection point $\left(a_{i} \cap b_{i}\right)$ on $S \times\left\{\frac{1}{2}\right\} \subset-\Sigma$ is an element in $\widehat{\mathrm{CFK}}(-\Sigma, \boldsymbol{\alpha}, \boldsymbol{\beta}, z, w)$ and the choice of $z$ assures that it is a cycle, hence it defines an element $\widehat{\mathscr{L}}(L)$ in $\widehat{\mathrm{HFK}}(-Y, L)$. As it was shown in [Lisca et al. 2008], the homology class $\widehat{\mathscr{L}}(L)$ is an invariant of the oriented Legendrian knot $L \subset(Y, \xi)$.

\section{Connection between the invariants}

Let $L$ be a Legendrian knot in a closed contact 3-manifold $(Y, \xi)$. The two invariants

$$
\operatorname{EH}(L)=\operatorname{EH}\left(Y-v(L),\left.\xi\right|_{Y-v(L)}\right) \in \operatorname{SFH}\left(-(Y-v(L)),-\Gamma_{\partial(Y-v(L))}\right),
$$

and $\widehat{\mathscr{L}}(L) \in \widehat{\mathrm{HFK}}(-Y, L)$ introduced above lie in two different groups, but if we change the suture on $\partial(Y-v(L))$ to two meridians $-m \cup m$ of $L$, the sutured Floer homology $\mathrm{SFH}(-(Y-v(L)),-m \cup m)$ can be identified with $\widehat{\mathrm{HFK}}(-Y, L)$. This modification of the suture can be achieved by attaching a basic slice to the sutured 3-manifold $Y-v(L)$, and according to [Honda et al. 2008] there is a map corresponding to this attachment. More generally:

Theorem 4.1 [Honda et al. 2008; see also Ghiggini and Honda 2008]. Suppose $\left(Y^{\prime}, \Gamma^{\prime}\right)$ is a balanced sutured submanifold of the balanced sutured 3-manifold $(Y, \Gamma)$ and all components of $Y-\operatorname{int}\left(Y^{\prime}\right)$ intersect $\partial Y$. Let $\xi$ be a contact structure on $Y-\operatorname{int}\left(Y^{\prime}\right)$ so that $\partial Y \cup \partial Y^{\prime}$ is convex with respect to $\xi$ and with dividing set $\Gamma \cup \Gamma^{\prime}$. Then there is a natural linear map

$$
\Phi_{\xi}: \operatorname{SFH}\left(-Y^{\prime},-\Gamma^{\prime}\right) \rightarrow \operatorname{SFH}(-Y,-\Gamma),
$$


induced by $\xi$. Moreover, if $Y^{\prime}$ is endowed with the contact structure $\xi^{\prime}$ such that $\Gamma_{\left(Y^{\prime}, \xi^{\prime}\right)}=\Gamma^{\prime}$ then

$$
\Phi_{\xi}\left(\mathrm{EH}\left(Y^{\prime}, \xi^{\prime}\right)\right)=\mathrm{EH}\left(Y, \xi^{\prime} \cup \xi\right) .
$$

We will apply this theorem in the special case when $\partial Y^{\prime}$ and $\partial Y$ are both 2-tori, $Y-\operatorname{int} Y^{\prime}=T^{2} \times[0,1]$ and the contact structure on the difference is a basic slice. The dividing set is given on $\partial\left(T^{2} \times[0,1]\right)$ by the dividing set of $\partial Y$ (on $T^{2} \times\{0\}$ ) and by the meridians of $L$ (on $T^{2} \times\{1\}$ ); there are two basic slices with the given boundary slopes. Notice that the attachment of the basic slice is actually equivalent to the attachment of a single bypass.

Trivialize $\partial(Y-v(L))$ with the meridian $m$ and the contact framing $l$, hence the dividing curves have slope $\infty$. The new dividing curve after attaching a bypass along any arc with slope between -1 and 0 has slope 0 . Up to isotopy there are only two different attachments (of opposite sign) depicted on Figure 6; these are the two different bypass attachments corresponding to the two different basic slices. These attaching curves together with the arcs of the dividing curves form an oriented curve on $\partial(Y-v(L))$; one of them represents $m$ the other one represents $-m$. Denote the former one by $c$.

Theorem 4.2. The map

$$
\Phi^{c}: \operatorname{SFH}\left(-(Y-v(L)),-\Gamma_{\partial(Y-v(L))}\right) \rightarrow \operatorname{SFH}(-(Y-v(L)),-m \cup m)
$$

induced by the basic slice attachment along $c$ maps $\mathrm{EH}(L)$ to the class which is identified with $\widehat{\mathscr{L}}(L)$ under the identification

$$
\Psi: \operatorname{SFH}(-(Y-v(L)),-m \cup m) \rightarrow \widehat{\operatorname{HFK}}(-Y, L) .
$$

Proof. Let $(S, g)$ be an open book for $(Y, \xi)$ that contains $L$ homologically essentially on one of its pages. Set $P=S-v_{S}(L)$ (where $v_{S}(L)$ denotes the tubular neighbourhood of $L$ in $S$ ) and $h=\left.g\right|_{P}$. We claim that the partial open book $(S, P, h)$ describes $\left(Y-v(L),\left.\xi\right|_{Y-v(L)}\right)$. Indeed, topologically the 3-manifold corresponding to this abstract partial open book is

$$
(S \times[-1,0] / \sim) \cup(P \times[0,1] / \sim),
$$
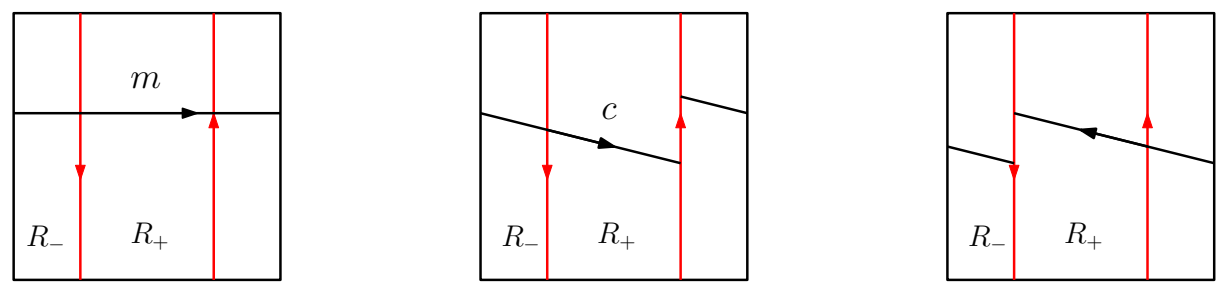

Figure 6. Bypass attachments to obtain meridians. 
which is equal to

$$
(S \times[-1,1] / \sim)-\left(v_{S}(L) \times[0,1]\right)=Y-v(L) .
$$

The contact structure on $S \times[-1,0] / \sim$ is the same, while on $P \times[0,1] / \sim$ (which is a subset of $S \times[0,1] / \sim)$ it is obviously tight. If we round the corners we get that the dividing curve is $\Gamma_{\partial(Y-v(L))}$, so the dividing curve on $P \times[0,1] / \sim$ must be $\partial P$.

Take a basis $\left\{b_{1}, \ldots, b_{k}\right\}$ of $S$ subordinated to $L$, such that $b_{1}$ is the halfmeridian of $L$. Then the left hand side of Figure 7 depicts the corresponding Heegaard diagram $\left(-\Sigma,\left\{\alpha_{1}, \ldots, \alpha_{k}\right\},\left\{\beta_{1}, \ldots, \beta_{k}\right\}, w, z\right)$ for $(-Y, L)$. Here $\Sigma=$ $S \times\left\{\frac{1}{2}\right\} \cup-S \times\left\{-\frac{1}{2}\right\}$ and the intersection point $\mathbf{x}=\left(a_{i} \cap b_{i}\right)_{i=1}^{k}$ represents the Legendrian invariant $\widehat{\mathscr{L}}(L)$ in $\widehat{\mathrm{HFK}}(-Y, L)$. The basis for $H_{1}(P, \partial S \cap \partial P)$ is $\left\{b_{2}, \ldots, b_{k}\right\}$ while the Heegaard surface is $-\widetilde{\Sigma}=P \times\left\{\frac{1}{2}\right\} \cup-S \times\left\{-\frac{1}{2}\right\}$. The corresponding Heegaard diagram for $\left(-(Y-v(L)),-\Gamma_{\partial(Y-v(L))}\right)$ is $\left(-\widetilde{\Sigma},\left\{\alpha_{2}, \ldots, \alpha_{k}\right\},\left\{\beta_{2}, \ldots, \beta_{k}\right\}\right)$ which is depicted on the right hand side of Figure 7. By definition $\mathbf{y}=\left(a_{i} \cap b_{i}\right)_{i=2}^{k}$ represents the contact invariant $\mathrm{EH}(L) \in \operatorname{SFH}\left(-(Y-v(L)),-\Gamma_{\partial(Y-v(L))}\right)$.

Attaching a bypass along $c$ changes the partial open book to $\left(S^{\prime}, P^{\prime}, h^{\prime}\right)$, where (with the notations described in Section 2D) $S^{\prime}=S \cup\left(1\right.$-handle) and $P^{\prime}=P \cup v\left(a_{+}\right)$. Note that $a_{+}$represents half of the meridian on $(\partial(\nu(L)))_{+} \subset S$, thus we can orient it. The 1-handle is attached to $S$ along $\partial S$ in the neighbourhood of the head of $a_{+}$ so that both of its feet are in the positive direction away from the head of $a_{+}$ with respect to the orientation of $\partial S$; see Figure 8. The monodromy remains the same restricted to $P$ (that is $\left.h^{\prime}\right|_{P}=h$ ) and as it was observed in Section 2D, $h^{\prime}\left(a_{+}\right)=a_{-}$and $a_{-}$splits as the core of the 1-handle and as $a_{-} \cap S$ which is isotopic to $c_{-}$. Note that $c_{-}$is a half-meridian of the knot $L$, thus the image of it on $S \times\left\{-\frac{1}{2}\right\}$ is isotopic to $g\left(a_{1}\right)$. Now we are ready to describe the Heegaard diagram $\left(-\left(\Sigma^{\prime},\left\{\alpha, \alpha_{2}^{\prime} \ldots, \alpha_{k}^{\prime}\right\},\left\{\beta, \beta_{2}^{\prime}, \ldots, \beta_{k}^{\prime}\right\}\right)\right)$ obtained from the partial open

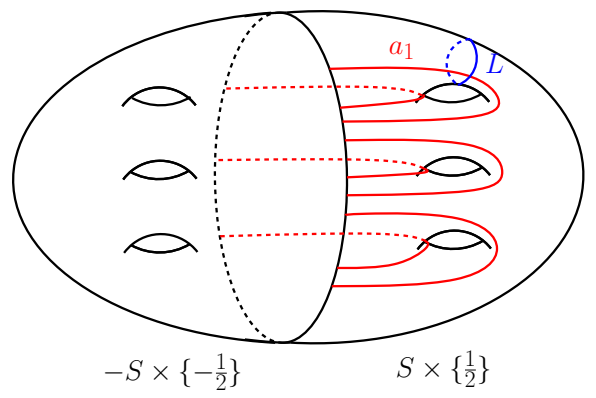

Heegaard diagram for $Y$

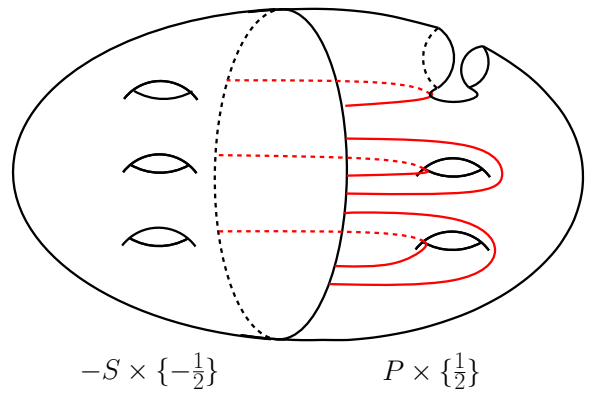

Heegaard diagram for $Y-N(L)$

Figure 7. Heegaard diagrams corresponding to the (partial) open books. 


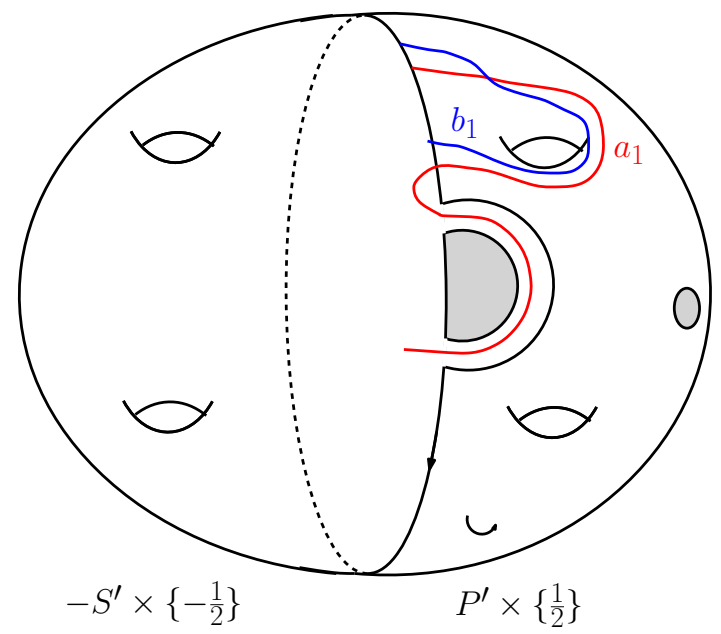

Figure 8. Heegaard diagram corresponding to $\left(S^{\prime}, P^{\prime}, h^{\prime}\right)$.

book $\left(S^{\prime}, P^{\prime}, h^{\prime}\right)$ in the usual manner. The Heegaard surface $-\Sigma^{\prime}$ is equal to $P^{\prime} \times$ $\left\{\frac{1}{2}\right\} \cup-S^{\prime} \times\left\{-\frac{1}{2}\right\}$, and the curves

$$
\beta^{\prime}=b_{+} \times\left\{\frac{1}{2}\right\} \cup b_{+} \times\left\{-\frac{1}{2}\right\} \quad \text { and } \quad \alpha^{\prime}=a_{+} \times\left\{\frac{1}{2}\right\} \cup a_{-} \times\left\{-\frac{1}{2}\right\},
$$

where $b_{+}$is the usual perturbation of $a_{+}$on $P^{\prime} . \quad \Sigma^{\prime}$ is obtained by gluing two surfaces together, each of which is diffeomorphic to $S-v$ (point). Indeed, the hole on the $S^{\prime}$-side comes from the 1-handle attachment. $P^{\prime}$ is just a union of the 1handles of $S$, thus the missing 2-handle gives us the other hole. This surface $\Sigma^{\prime}$ is thus diffeomorphic to $\Sigma-v(z)-v(w)$, where we think of $v(z)$ being deleted from the $S^{\prime}$ - and $v(w)$ from the $P^{\prime}$-side. Under this identification $b_{+}$(and thus $a_{+}$) is isotopic to $b_{1}$ on $P^{\prime}$, hence $\beta^{\prime}=b_{+} \times\left\{\frac{1}{2}\right\} \cup b_{+} \times\left\{-\frac{1}{2}\right\}$ and $\beta_{1}$ are isotopic on $\Sigma^{\prime}$. Recall that $h^{\prime}\left(a_{+}\right)$on $S^{\prime} \times\left\{-\frac{1}{2}\right\}$ was isotopic to the union of $g\left(a_{1}\right)$ and the core of the 1-handle. So $\alpha^{\prime}$ is isotopic to $\alpha_{1}$ on $\Sigma-v(z)$. The core part of $h^{\prime}\left(a_{+}\right)$makes $\alpha^{\prime}$ and $\beta^{\prime}$ to go around the hole $v(w)$ from different sides, thus $\alpha^{\prime}$ is isotopic to $\alpha_{1}$ on $\Sigma^{\prime}$. In conclusion, the Heegaard diagram $\left(-\Sigma^{\prime},\left\{\alpha^{\prime}, \alpha_{2}, \ldots, \alpha_{k}\right\},\left\{\beta^{\prime}, \beta_{2}, \ldots, \beta_{k}\right\}\right)$ is isotopic to $\left(-(\Sigma-v(z \cup w)),\left\{\alpha_{1}, \ldots, \alpha_{k}\right\},\left\{\beta_{1}, \ldots, \beta_{k}\right\}\right)$. The contact invariant $\mathrm{EH}(L)$ is mapped to the contact invariant $\mathrm{EH}(Y-v(L),-m \cup m)$ under the map induced by the basic slice, and thus it represents the Legendrian invariant in $\widehat{\mathrm{CFK}}(-\Sigma, \boldsymbol{\alpha}, \boldsymbol{\beta}, z, w)$, which proves the statement.

Proof of Theorem 1.1. With the identifications above, the proof of Theorem 1.1 is now complete.

Next we turn to the proof of the remaining statements described in Section 1. 
Proof of Theorem 1.5. Take a standard contact neighbourhood $v(L)$ of $L$ and stabilize $L$ inside it. Then $L^{ \pm}$has a standard contact neighbourhood $v\left(L^{ \pm}\right) \subset v(L)$. The contact manifold $\left(v(L)-v\left(L^{ \pm}\right),\left.\xi\right|_{v(L)-v\left(L^{ \pm}\right)}\right)$, as it is explained in [Etnyre and Honda 2001], is a basic slice, that is, $Y-v\left(L^{ \pm}\right)$is obtained from $Y-v(L)$ by a bypass attachment. We can view $Y-v(L)$ as the result of a bypass attachment to the boundary of $Y-v\left(L^{ \pm}\right)$from the back. As usual, the two basic slices with the above boundary conditions have opposite relative Euler classes. To figure out which one corresponds to the positive and which one to the negative stabilization we first examine a model case. (For a related discussion see [Etnyre and Honda 2001].) Suppose that $\operatorname{tb}(L)<0$ and take a Seifert surface $S$ for $L$, giving rise to the Seifert surface $S^{p}$ (respectively $S^{m}$ ) for $L^{+}$(respectively $L^{-}$). These surfaces are oriented such that their boundary orientations give the orientations for the knot. By $\operatorname{tb}(L)<0$ we can assume that $S$ is in convex position. We have $\operatorname{tb}\left(L^{ \pm}\right)=\operatorname{tb}(L)-1$, thus the dividing curve hits the boundary of the Seifert surface $S$ in $2|\operatorname{tb}(L)-1|$ points. In the collar neighbourhood of the boundary (diffeomorphic to $S^{1} \times I$ ), the dividing curves of $S$ are the line segments $k \frac{2 \pi}{2|\operatorname{tb}(L)|} \times I$ where $0 \leq k<2|\operatorname{tb}(L)|$. Once again, by the negativity of $\operatorname{tb}(L)$ the bypass attachment corresponds to the gluing of an annulus to the boundary of $S$ with dividing curves $k \frac{2 \pi}{2|\operatorname{tb}(L)|} \times I(0 \leq$ $k<2|\operatorname{tb}(L)|)$ and a boundary parallel curve that is disjoint from the others. This boundary parallel curve bounds a domain; see Figure 9 . The rotation numbers are $\operatorname{rot}\left(L^{ \pm}\right)=\operatorname{rot}(L) \pm 1$, thus by the formula $\operatorname{rot}(S)=\chi\left(S_{+}\right)-\chi\left(S_{-}\right)$we get that the extra domain on $S^{p}$ (on $S^{m}$, respectively) is in the positive (respectively negative) region. Using edge rounding we get that the attaching curve corresponding to the positive (respectively negative) stabilization must end in the positive (respectively negative) region with respect to the orientation of the knot. The left hand side of Figure 10 depicts the arc $p$ (and $n$, respectively) along which the bypass has to be attached (from the back) to obtain $Y-v(L)$.

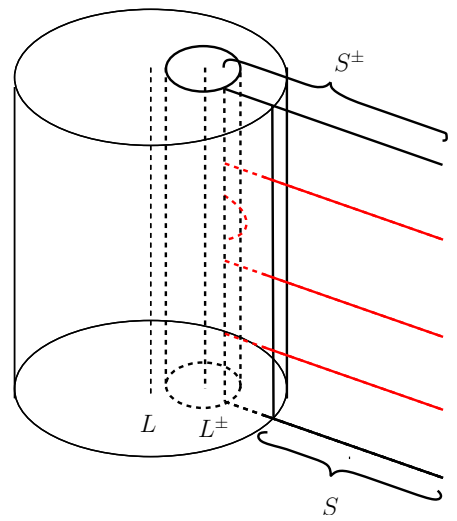

Figure 9. Neighbourhood of a Legendrian knot and its stabilization. 


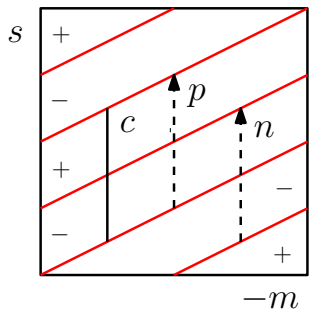

$\partial\left(Y-\nu\left(L^{ \pm}\right)\right)$

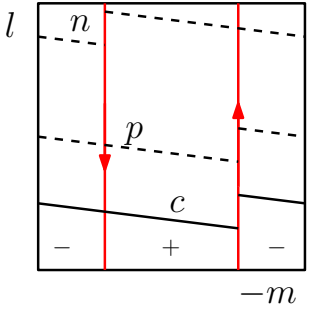

$\partial\left(Y-\nu\left(L^{ \pm}\right)\right)$

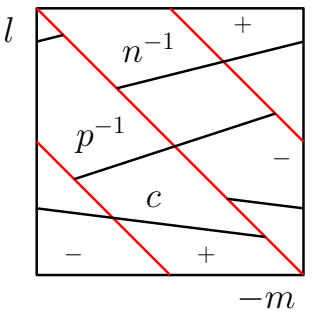

$\partial(Y-\nu(L))$

Figure 10. Attaching curves for the bypasses corresponding to the stabilizations. The dashed line indicates that the bypass is attached from the back. On the left-hand picture $s$ denotes the Seifert framing of the knot, while on the two right-hand pictures $l$ is given by the contact framing of the Legendrian knot.

Both the stabilization and the bypass attachment are local operations, thus the above described phenomenon remains true for any Legendrian knot (without the assumption $\operatorname{tb}(L)<0)$. The arcs $p$ and $n$ have the same slope, but they end in regions of different sign. Consider the middle diagram of Figure 10 for the general picture for $T^{2}$, trivialized by the meridian $m$ and the Thurston-Bennequin framing $l$.

By Theorem 4.1 the map corresponding to the bypass attachment maps $\mathrm{EH}(L)$ to $\operatorname{EH}\left(L^{ \pm}\right)$. To get $\widehat{\mathscr{L}}\left(L^{ \pm}\right)$we need to attach another bypass, so that the new dividing curves are meridians, hence this second bypass is attached along the $\operatorname{arc} c$.

In the case of positive stabilization, the manifold

$$
\left(Y-v\left(L^{+}\right),\left(\left.\xi\right|_{Y-v\left(L^{+}\right)}\right)^{c}\right)=\left(Y-v(L),\left(\left.\xi\right|_{Y-v(L)}\right)^{p^{-1} c}\right)
$$

is overtwisted. Indeed, performing the positive stabilization first one can indicate both bypasses in one picture, one attached from the back: $p^{-1}$ drawn by dashed line in the middle picture of Figure 10 and $c$ from the front. These curves are parallel, thus the corresponding bypasses ("half overtwisted disks") form an overtwisted disk in $\left(Y-v(L),\left(\left.\xi\right|_{Y-v(L)}\right)^{p^{-1} c}\right)$. It is known that the sutured invariant of an overtwisted structure vanishes [Honda et al. 2007, Corollary 4.3.], therefore so does $\widehat{\mathscr{L}}\left(L^{+}\right)$.

In the case of negative stabilization, the contact structure $\left(T^{2} \times I, \xi^{n^{-1} c}\right)$ is universally tight. This can be seen by first passing to $\partial(Y-v(L))$ (see the right hand side of Figure 10) and then noting that the two bypasses attached there are of the same sign, so they do not induce an overtwisted disk. The union of the two basic slices is minimally twisting, and in this case the range of slopes is $[0, \infty]=[0,1] \cup[1, \infty]$. Therefore the result is still a basic slice, thus the composition of the two bypass attachments along $n$ and $c$ is equivalent to a single 
bypass attachment along $c$. This immediately implies $\widehat{\mathscr{L}}(L)=\widehat{\mathscr{L}}\left(L^{-}\right)$, concluding the proof.

Next we turn to the proof of the statement concerning the vanishing of the Legendrian invariant in the presence of Giroux torsion. We start by recalling Giroux torsion.

Definition 4.3. The contact structure $\xi_{n}$ on $T^{2} \times[0,1]=\mathbb{R} / \mathbb{Z} \times \mathbb{R} / \mathbb{Z} \times[0,1]=$ $\{(x, y, z))\}$ is defined by

$$
\xi_{n}=\operatorname{ker}(\cos (2 \pi n z) d x-\sin (2 \pi n z) d y) .
$$

A (not necessarily closed) contact 3-manifold $(Y, \xi)$ has Giroux torsion $\tau(Y, \xi) \geq$ $n$ if it contains an embedded submanifold $T^{2} \times I$ with the property that $\left(T^{2} \times\right.$ $\left.I,\left.\xi\right|_{T^{2} \times I}\right)$ is contactomorphic to $\left(T^{2} \times[0,1], \xi_{n}\right)$.

Proof of Corollary 1.2. The proof is a simple adaptation of the proof for the closed case given by Ghiggini, Honda, and Van Horn-Morris [2007]. As ( $Y-$ $\left.v(L), \xi_{Y-v(L)}\right)$ has positive Giroux torsion, there is a submanifold $T^{2} \times I$, such that $\left.\xi\right|_{T^{2} \times I}=\xi_{n}$ for some $n>0$. It was shown in [Ghiggini et al. 2007] that $\mathrm{EH}\left(T^{2} \times I, \xi_{n}\right)=0$.

The application of Theorem 4.1 for the contact 3-manifold pair $\left(Y-v(L), T^{2} \times\right.$ $[0,1])$ provides a map

$$
\operatorname{SFH}\left(-\left(T^{2} \times I\right),-\Gamma_{\partial\left(T^{2} \times I\right)}\right) \rightarrow \operatorname{SFH}\left(-(Y-v(L)),-\Gamma_{Y-v(L)}\right)
$$

mapping the contact element $\mathrm{EH}\left(T^{2} \times I, \xi_{n}\right)=0$ to the contact element $\mathrm{EH}(L)=$ $\mathrm{EH}\left(Y-v(L),\left.\xi\right|_{Y-v(L)}\right)$. This implies that $\operatorname{EH}(L)=0$, hence in the light of Theorem 1.1 we get that $\widehat{\mathscr{L}}(L)=0$, concluding the proof.

Proof of Theorem 1.7. As in the proof of Theorem 1.1, we attach a bypass along the arc $e$ of Figure 11 and change the dividing curve on the torus boundary to $\Gamma_{\partial(Y-v(L))}^{e}$

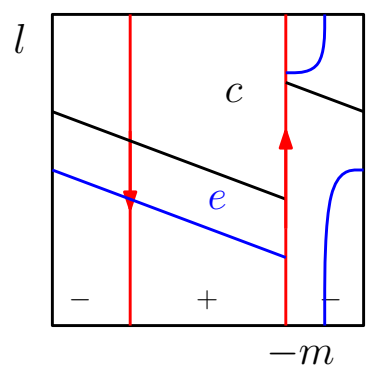

Figure 11. Attaching curves for the bypasses on $\partial(Y-v(L))$ to obtain dividing curves of slope 1 . 
of slope -1 . There are two choices for such arcs, but again the orientation of $L$ assigns the one depicted on Figure 11.

This bypass attachment gives rise to a map

$$
\Phi^{e}: \operatorname{SFH}\left(-(Y-v(L)),-\Gamma_{\partial(Y-v(L))}\right) \rightarrow \operatorname{SFH}\left(-(Y-v(L)),-\Gamma_{\partial(Y-v(L))}^{e}\right) .
$$

By filling the boundary with a solid torus, the latter homology is identified with $\widehat{\mathrm{HFK}}\left(-Y_{-1}, L^{\prime}\right)$. Denote the composition of the above maps by

$$
G: \operatorname{SFH}\left(-(Y-v(L)),-\Gamma_{\partial(Y-v(L))}\right) \rightarrow \widehat{\operatorname{HFK}}\left(-Y_{-1}, L^{\prime}\right) .
$$

We claim that the homomorphism $G$ maps $\operatorname{EH}(L)$ to $\widehat{\mathscr{L}}\left(L^{\prime}\right)$. Indeed, consider an open book $(S, h)$ adapted to $(Y, \xi, L)$. The same open book is adapted to $\left(Y_{-1}, \xi_{-1}, L^{\prime}\right)$, with the only difference in the monodromy: the monodromy $h^{\prime}$ for the latter triple is multiplied by a right-handed Dehn twist along $L$; see [Ozbagci and Stipsicz 2004, page 133]. Using the notations introduced in Section 2, the map $G$ corresponds to changing the partial open book $\left(S, P=S-v_{S}(L),\left.h\right|_{P}\right)$ to $\left(S^{\prime}, P^{\prime}, h^{\prime \prime}\right)$ corresponding to the bypass attachment. The image of the halfmeridian $a_{+}$under $h^{\prime \prime}$ is $h\left(a^{+}\right)$multiplied by a right-handed Dehn twist along $L$. Therefore $G(\mathrm{EH}(L))=\widehat{\mathscr{L}}\left(L^{\prime}\right)$.

After attaching the bypass along $e$, we can apply another bypass attachment along $c$ of Figure 11 to obtain the meridian as dividing curve. We have already seen in the proof of Theorem 1.5 that the composition of these two bypasses is a basic slice; thus we have the commutative diagram

$$
\begin{gathered}
\operatorname{SFH}\left(-(Y-v(L)),-\Gamma_{\partial(Y-v(L))}\right) \\
\operatorname{SFH}(-(Y-v(L)),-m \cup m)=\widehat{\operatorname{HFK}}(-Y, L)
\end{gathered}
$$

The maps in this triangle map the contact invariants as

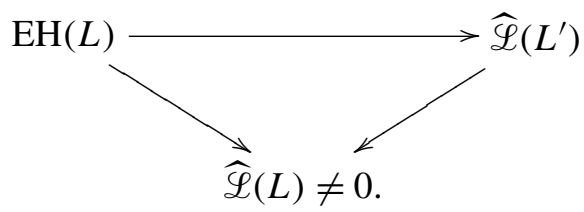

Therefore $\widehat{\mathscr{L}}\left(L^{\prime}\right)$ does not vanish, concluding the proof.

\section{Acknowledgements}

We would like to thank Ko Honda and Paolo Ghiggini for helpful discussions. 


\section{References}

[Chekanov 2002] Y. Chekanov, "Differential algebra of Legendrian links", Invent. Math. 150:3 (2002), 441-483. MR 2003m:53153 Zbl 1029.57011

[Eliashberg 1998] Y. Eliashberg, "Invariants in contact topology", Doc. Math. Extra Vol. II (1998), 327-338. MR 2000a:57068 Zbl 0913.53010

[Eliashberg and Fraser 1998] Y. Eliashberg and M. Fraser, "Classification of topologically trivial Legendrian knots", pp. 17-51 in Geometry, topology, and dynamics (Montreal, 1995), edited by F. Lalonde, CRM Proc. Lecture Notes 15, Amer. Math. Soc., Providence, RI, 1998. MR 99f:57007 Zbl 0907.53021

[Eliashberg and Fraser 2008] Y. Eliashberg and M. Fraser, "Topologically trivial Legendrian knots”, 2008. arXiv 0801.2553

[Etgü and Ozbagci 2007] T. Etgü and B. Ozbagci, "Partial open book decompositions and the contact class in sutured Floer homology", preprint, 2007. arXiv 0711.0880

[Etgü and Ozbagci 2008] T. Etgü and B. Ozbagci, "Relative Giroux correspondence", preprint, 2008. arXiv 0802.0810

[Etnyre 2005] J. B. Etnyre, "Legendrian and transversal knots", pp. 105-185 in Handbook of knot theory, edited by W. Menasco and M. Thistlethwaite, Elsevier B. V., Amsterdam, 2005. MR 2006j: $57050 \mathrm{Zbl} 1095.57006$

[Etnyre 2008] J. Etnyre, 2008. Private communication.

[Etnyre and Honda 2001] J. B. Etnyre and K. Honda, "Knots and contact geometry. I. Torus knots and the figure eight knot”, J. Symplectic Geom. 1:1 (2001), 63-120. MR 2004d:57032 Zbl 1037. 57021

[Ghiggini and Honda 2008] P. Ghiggini and K. Honda, "Giroux torsion and twisted coefficients", preprint, 2008. arXiv 0804.1568

[Ghiggini et al. 2007] P. Ghiggini, K. Honda, and J. Van Horn-Morris, "The vanishing of the contact invariant in the presence of torsion", preprint, 2007. arXiv 0706.1602

[Honda 2000] K. Honda, "On the classification of tight contact structures. I", Geom. Topol. 4 (2000), 309-368. MR 2001i:53148 Zbl 0980.57010

[Honda et al. 2007] K. Honda, W. Kazez, and G. Matić, "The contact invariant in sutured Floer homology”, preprint, 2007. arXiv 0705.2828

[Honda et al. 2008] K. Honda, W. Kazez, and G. Matić, "Contact structures, sutured Floer homology and TQFT”, preprint, 2008. arXiv 0807.2431

[Juhász 2006] A. Juhász, "Holomorphic discs and sutured manifolds", Algebr. Geom. Topol. 6 (2006), 1429-1457. MR 2007g:57024 Zbl 1129.57039

[Lisca et al. 2008] P. Lisca, P. Ozsváth, A. Stipsicz, and Z. Szabó, "Heegaard Floer invariants of Legendrian knots in contact 3-manifolds", preprint, 2008. arXiv 0802.0628

[Ng et al. 2007] L. Ng, P. Ozsváth, and D. Thurston, "Transverse knots distinguished by knot Floer homology", preprint, 2007. arXiv 0703446

[Ozbagci and Stipsicz 2004] B. Ozbagci and A. I. Stipsicz, Surgery on contact 3-manifolds and Stein surfaces, Bolyai Society Mathematical Studies 13, Springer, Berlin, 2004. MR 2005k:53171 Zbl 1067.57024

[Ozsváth and Stipsicz 2008] P. Ozsváth and A. I. Stipsicz, "Contact surgeries and the transverse invariant in knot Floer homology", preprint, 2008. arXiv 0803.1252 
[Ozsváth and Szabó 2004a] P. Ozsváth and Z. Szabó, "Holomorphic disks and knot invariants", Adv. Math. 186:1 (2004), 58-116. MR 2005e:57044 Zbl 1062.57019

[Ozsváth and Szabó 2004b] P. Ozsváth and Z. Szabó, "Holomorphic disks and three-manifold invariants: properties and applications", Ann. of Math. (2) 159:3 (2004), 1159-1245. MR 2006b:57017 Zbl 1081.57013

[Ozsváth and Szabó 2004c] P. Ozsváth and Z. Szabó, "Holomorphic disks and topological invariants for closed three-manifolds", Ann. of Math. (2) 159:3 (2004), 1027-1158. MR 2006b:57016 Zbl 1073.57009

[Ozsváth et al. 2008] P. Ozsváth, Z. Szabó, and D. Thurston, "Legendrian knots, transverse knots and combinatorial Floer homology”, Geom. Topol. 12:2 (2008), 941-980. MR 2403802

[Rasmussen 2003] J. Rasmussen, Floer homology and knot complements, PhD thesis, Harvard University, 2003. arXiv 0607691

[Torisu 2000] I. Torisu, "Convex contact structures and fibered links in 3-manifolds", Internat. Math. Res. Notices 9 (2000), 441-454. MR 2001i:57039 Zbl 0978.53133

[Vela-Vick 2008] D. S. Vela-Vick, "On the transverse invariant for bindings of open books", preprint, 2008. arXiv 0806.1729

Received June 9, 2008.

ANDRÁS I. STIPSICZ

RÉNYi INSTITUTE OF MATHEMATICS

HUNGARIAN ACADEMY OF SCIENCES

REALTANODA UTCA 13-15 H-1053 BUDAPEST

HUNGARY

stipsicz@ renyi.hu

http://www.renyi.hu/ stipsicz

VERA VÉRTESI

INSTITUTE OF MATHEMATICS

EÖTVÖS LORÁND UNIVERSITY

PÁZMÁNY PÉTER SÉTÁNY 1/C

H-1117 BUDAPEST

HUNGARY

wera@szit.bme.hu

www.szit.bme.hu/ wera 